\title{
CLL and Lymphoma Studies at EHA2021 and 16-ICML
}

\section{DOI: 10.36000/hbT.OH.2021.09.051}

Rossi D. Personal highlights: CLL and lymphoma studies at EHA2021 and 16-ICML. healthbook TIMES Onco Hema. 2021;(9):50-51.

Prof. Dr Davide Rossi, MD, PhD

Deputy Head of the Division of Hematology

Oncology Institute of Southern Switzerland (IOSI)

Bellinzona, Switzerland

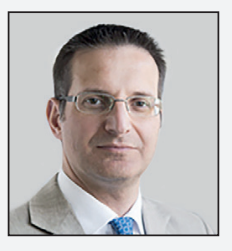

At EHA2021 and 16-ICML, there were several relevant presentations in the field of chronic lymphocytic leukemia (CLL) and Iymphoma. The first reported on the primary analysis of GLOW, a randomized, phase III trial comparing the time-limited treatment with ibrutinib plus venetoclax, a novel oral combination, versus chlorambucil plus obinutuzumab in treatment-naïve, frail CLL patients. ${ }^{1}$ The trial was positive, with a significant improvement in progression-free survival (PFS) with ibrutinib plus venetoclax (median, not reached [NR] vs 21.0 months; HR: 0.216 [95\% Cl: 0.131-0.357]; $p<0.0001$ ), despite a relatively short follow-up. The treatment was deemed to be tolerated in this population of older and unfit CLL patients.

The other two studies compared two second-generation Bruton's tyrosine kinase (BTK) inhibitors, zanubrutinib ${ }^{2}$ and acalabrutinib ${ }^{3,4}$, respectively, with ibrutinib in patients with relapsed CLL. The main takeaway message from these trials is that second-generation BTK inhibitors are better tolerated than ibrutinib, especially in terms of lower rates of cardiac events, which may occur in a fraction of patients treated with ibrutinib. Furthermore, the biomarker analyses of the CLL14 trial have started defining high-risk patients who might not benefit from the combination treatment of venetoclax plus obinutuzumab. 5,6 Although results indicated that this time-limited treatment might not be as advantageous for CLL patients with TP53 abnormalities compared with those without these aberrations (median PFS, 49.0 months vs NR), data to draw final conclusions are awaited from the longer follow-up from this trial, as well as from head-to-head studies like CLL177.

Furthermore, various interesting trials presented at the congresses assessed immunotherapy in lymphomas. Of these, ZUMA- $5^{8}$ and ELARA 9,10 investigated chimeric antigen receptor (CAR) T-cell therapies, axicabtagene ciloleucel and tisagenlecleucel, respectively, 
